\title{
Una aproximación rusa al poder blando en el actual sistema internacional
}

Carmen Scocozza*

RESUMEN

El artículo analiza la creciente centralidad de las políticas de poder blando en la Rusia de Putin. Sin embargo, a pesar de las declaraciones sobre la importancia del ejercicio de un poder alternativo a la mera fuerza militar, se destacan las dificultades de Moscú que, en los momentos de crisis internacionales, regresa a la tradicional política imperial frustrando los esfuerzos hechos para promover una imagen positiva del país. Por tanto, el principal problema para la Federación Rusa parece ser su capacidad de resultar creíble y de definir un sistema de valores atractivo, que no dependa de las directivas gubernamentales y sea el resultado de principios compartidos en una sociedad madura.
Palabras clave: Rusia, poder blando, poder duro, CEI.

\section{A Russian approach to soft power in the current international system}

\begin{abstract}
The article analyzes the increasing centrality of soft power policies in Putin's Russia. Despite assertions about the importance of using power alternative to mere military force, Moscow's difficulties in doing so is evident, as it returns to traditional imperial policy in times of international crisis, and thereby
\end{abstract}

* PhD y posdoctorado en Historia y Política. Docente Maestría en Ciencia Política, Universidad Católica de Colombia en convenio con la Università degli Studi di Salerno (Italia). [cscocozza@ucatolica.edu.co]

Recibido: 15 de febrero de 2016/ Modificado: 19 de septiembre de 2016/ Aceptado: 25 de septiembre de 2016 Para citar este artículo

Scocozza, C. (2017). Una aproximación rusa al poder blando en el actual sistema internacional. OASIs, 25 , 63-74. DOI: https://doi.org/10.18601/16577558.n25.04 
thwarts its efforts to promote a positive image of the country. Therefore, the main problem for the Russian Federation is its ability to be credible and to define a set of attractive values which do not depend on government directives, but that come about as a result of shared principles in a mature society.

Key words: Russia, soft power, hard power, CIS.

\section{INTRODUCCIÓN}

El fin de la contraposición bipolar, junto a la redefinición de un sistema internacional caracterizado por diferentes centros de poder, ha suscitado en la mayoría de los Estados una oportuna reflexión sobre la necesidad de encontrar nuevos instrumentos para atraer potenciales amigos y afirmar su visión de las relaciones internacionales.

En un contexto global, mucho más fluido y dinámico, el recurso a la fuerza militar tendría que representar la última opción respecto a otras posibilidades para conseguir nuevos socios, por ejemplo, presentándose como un modelo exitoso y atractivo. En otras palabras, en el actual sistema internacional se destaca la importancia creciente de un poder blando que, en la definición de Joseph Nye, es representado por todos los recursos -como la cultura política, los valores sociales, las normas morales y el carisma cultural- en poder de un país, que permiten influir sobre las preferencias de otros Estados y determinar un acercamiento a su propio sistema de referencia (1990, p. 168).
Si, en los últimos ańos, este tipo de poder ha sido utilizado por varios países para alcanzar sus objetivos de política exterior, también en Rusia se han empezado a desarrollar nuevas estrategias dirigidas a relanzar una imagen demasiado comprometida con un pasado de opresión imperial.

Sin embargo, hablar de soft power y de Rusia puede parecer un poco arriesgado, en la medida en que, en el imaginario colectivo, la política del Kremlin se ha caracterizado siempre por un poder duro, ejercido a través de la coerción y la fuerza militar. De todas formas, al considerar la tendencia de los más recientes documentos por ampliar el concepto de soft power, incluyendo entre sus indicadores fundamentales la economía, el capital humano, la política, la cultura y la diplomacia, se puede afirmar que en la Federación Rusa se encuentra algo similar (Liu y Guan, 2014, p. 75).

De hecho, no se puede no considerar una tradición milenaria y la importancia de raíces históricas y culturales que, desde la época zarista, han permitido al imperio ruso presentarse como el representante de una civilización autónoma y el país guía de todos los pueblos eslavos. Aún más, se señala una forma de poder blando en el periodo soviético, cuando se definieron diferentes estrategias de atracción ideológica y promoción de Unión Soviética para cooptar nuevos aliados en la competición entre modelos sociales, políticos y económicos contrapuestos.

Por otro lado, la repentina caída de la URss, y la dificultad en el proceso de transición, han representado un obstáculo al desarrollo de una verdadera política de soft 
power en el momento en que el Gobierno tenía que enfrentarse con la destrucción de su patrimonio cultural, el deterioro de su imagen a nivel interno e internacional, y la pérdida consiguiente de fuerza y credibilidad. Solo en tiempos más recientes, con la llegada de Putin al Kremlin, se ha registrado una más clara voluntad de restaurar una política independiente y autónoma, reafirmando las prioridades nacionales y regresando al escenario mundial como un actor capaz de exigir el respeto debido.

Por tanto, después de un primer momento necesario a la prioritaria reorganización del hard power en el país, también en Rusia se ha comenzado a mostrar en los últimos años una cierta sensibilidad respecto a la implementación de una política de poder blando que, como demuestra el caso más significativo de Estados Unidos, permite alcanzar importantes objetivos y obtener considerables ventajas (Tsygankov, 2006).

\section{LA PRESIDENCIA DE PUTIN Y LAS POLÍTICAS DE SOFT POWER}

Consciente de esto, ya en la víspera de las elecciones presidenciales del marzo de 2012, Putin publicó en la revista Moskovskie Novosti lo que puede ser considerado el documento programático sobre los objetivos estratégicos de un país que necesitaba adaptarse "a un mundo que cambia”. De hecho, frente a la denuncia de la erosión del derecho internacional y a la injerencia occidental que debilita "el principio secular de la soberanía nacional" (Putin, 2012a), Rusia buscaba posicionarse de nuevo en un sistema mundial atravesado por profundas crisis económicas y políticas. En un momento en que las relaciones con Occidente aún no habían tocado el punto más crítico de la guerra en Ucrania, Putin manifestaba así una general voluntad de diálogo y cooperación para reforzar la seguridad global.

Es interesante observar cómo el entonces primer ministro ruso, mientras que criticaba la interferencia occidental en los países árabes y los intentos de exportar la democracia a través de la fuerza ${ }^{1}$, ponía al mismo tiempo al centro de su reflexión el peligro de un soft power visto como "un conjunto de instrumentos y métodos para alcanzar objetivos de política exterior sin la utilización de armas, sino a través de medios de información y otros tipos de influencia" (Putin, 2012a).

Según Putin, esta conducta terminaba por favorecer "la manipulación de la conciencia pública y una injerencia directa en la política interna de los Estados soberanos". Más en general, lo que se puede destacar en este artículo es que la postura en contra de la utilización de estas herramientas demuestra, al mismo tiempo, la creciente atención respecto a diferentes acciones de política exterior. De este modo, si por un lado Putin criticaba a Occidente por el uso desprejuiciado de los

\footnotetext{
1 Recordamos que el artículo de Putin se publica en un momento particular, tras las manifestaciones y protestas conocidas como "primavera árabe” que, desde la revolución tunecina de 2010, habían cruzado diferentes países del mundo árabe, y después de la intervención de la OTAN en Libia.
} 
instrumentos de control de la opinión pública, por el otro daba claras indicaciones sobre las nuevas directrices de la política exterior rusa. En particular, expresaba la voluntad de Moscú de ir más allá de los tradicionales pilares de una política basada en la fuerza militar y en la capacidad de atracción de su fuerza económica. Frente a la existencia de prejuicios en contra de Rusia, y a la falta de reconocimiento de su contribución al desarrollo de la civilización mundial, el artículo terminaba con el augurio de una mayor inversión en la cultura y en la información para difundir una imagen más objetiva y positiva de su país (Putin, 2012a).

Indudablemente, la posición de Putin era coherente con la idea general de que, en el actual sistema internacional policéntrico, el principal desafío para una política exterior competitiva está en la exigencia de buscar una visión más amplia y nuevos expedientes para incrementar el número de los países amigos.

Después de años caracterizados por la pérdida de su capacidad de atracción, tanto a nivel cultural como ideológico, Moscú regresa entonces al escenario internacional convencido de la necesidad de proponerse como un modelo exitoso para recuperar los vínculos con los viejos países soviéticos y ampliar las colaboraciones con otros actores.

Por esta razón, en 2012, el entonces neoelegido presidente Putin puso en el orden del día de la agenda política rusa la promoción del "poder blando" (mjagkaja sila) y, con ocasión del encuentro de los embajadores de la Federación Rusa, en julio de ese mismo año, reiteró la importancia de complementar los tradicionales métodos diplomáticos con nuevas estrategias para la defensa de los intereses nacionales y la proyección hacia afuera de una nueva Rusia, y de su importante herencia cultural, intelectual y espiritual (Putin, 2012b).

El poder blando se convierte, además, en un aspecto central del nuevo "Concepto de política exterior" de Rusia, de 12 de febrero de 2013. En el documento se señala que el principal objetivo de la actividad internacional rusa es la creación de un contexto mundial estable y de condiciones favorables para el crecimiento económico y el desarrollo del país. Es interesante observar que, según la opinión del Kremlin, la estabilidad y la seguridad mundial parecen amenazadas por lo que puede ser definido "un abuso de poder blando" por parte de varios actores internacionales. Se manifiesta, entonces, una contradicción de la postura rusa frente al soft power. Por una parte, reconoce que estas herramientas tecnológicas, culturales e informativas representan "un componente indispensable de la actual política internacional"; por otra, denuncia los países que han convertido estos instrumentos en pilares fundantes de sus políticas exteriores (Putin, 2013). Probablemente, esta ambivalencia es determinada por la creciente percepción de la importancia de estos expedientes de política exterior y la conciencia de tener todavía mucho qué hacer en este ámbito para llegar a los resultados logrados por Occidente.

En un contexto de fuerte competencia global, en el documento se denuncia el peligro de un uso "destructivo e ilegal" de este tipo de poder convertido, en la mayoría de los casos, en una injerencia indebida en los asuntos de los Estados soberanos, en la desestabilización de las políticas internas y en la manipulación de la opinión pública a través del financiamiento 
de proyectos supuestamente humanitarios y culturales.

A pesar de las fuertes críticas, el concepto de política exterior de 2013 pone entre los objetivos prioritarios la implementación del poder blando para promover "una imagen positiva que corresponda al alto estatus de su cultura, de la educación, de la ciencia, del deporte, del nivel de desarrollo de la sociedad civil” del país (Putin, 2013). Es innegable, por tanto, la renovada atención respecto a esta forma de poder; que sea rechazada o admirada, sin duda destaca la centralidad de un tema casi desconocido hasta hace poco años.

\section{LA IMPORTANCIA DEL “EXTRANJERO CERCANO"}

La razón principal de esta formalización oficial se encuentra en los acontecimientos internacionales que han obligado a Rusia a reconsiderar las directrices de su política exterior y a reflexionar sobre las causas de la erosión de su influencia, en particular en el espacio possoviético. Por ejemplo, no se puede omitir señalar el fuerte impacto que han tenido en Rusia "las revoluciones de colores", es decir, las movilizaciones políticas que se dieron entre 2003 y 2005, en Georgia, Ucrania y Kirguistán, las cuales han demostrado la capacidad de atracción del modelo occidental también en territorios considerados históricamente de pertenencia rusa (Ó Beacháin y Polese, 2012).

En el momento en que Moscú asistía en estos países a debates acalorados, y a la formación de movimientos en favor de Occidente, empezó a hacer autocrítica respecto a la ausencia de un mensaje ruso unívoco y claro; de hecho, el vacío ideal que se generó después de la caída de la Unión Soviética ha representado una imperdible oportunidad para todos los que han sabido aprovechar y llenar el espacio que quedó libre.

Rusia parte del convencimiento de que fortalecer los vínculos en el "extranjero cercano” -así como Moscú define todo el ex espacio soviético- es un interés tanto del Kremlin como de los nuevos Estados, y que la distancia de los últimos años haya sido culpa de una falta de comunicación y de la dificultad de explicar las razones de la presencia rusa en la región.

Si las relaciones con estos países siguen siendo una prioridad para el Gobierno ruso, la necesidad de ofrecer "una alternativa ideológica" al modelo occidental se ha convertido en el principal objetivo de una política exterior más eficaz. Además, el logro de este objetivo se considera el punto de partida de cualquier otra estrategia para consolidar el prestigio del Kremlin en el escenario mundial (Karabeshkin y Sergunin, 2015).

La pregunta de la cual se parte es: ¿cómo ha sido posible una tal dispersión en una zona caracterizada por el mismo trasfondo histórico y cultural? Para los rusos el problema fundamental ha sido el inteligente ejercicio de poder blando a través de los medios de comunicación y la presencia de organizaciones no gubernamentales que han permitido a Occidente ganar terreno en el espacio eurasiático. Por esta razón, se ha empezado a manifestar la voluntad de "desarrollar una eficaz infraestructura de ideas, instituciones, redes y medios de comunicación [...] para recuperar su propia influencia no solo a nivel gubernamental, sino a nivel de las sociedades" 
(Krastev, 2005), y competir con los países occidentales en el mismo campo.

El debate se ha enfocado, entonces, en la oportunidad de encontrar una "idea movilizadora" para reaccionar a la progresiva influencia occidental y convencer a los viejos aliados de que "Rusia no combatirá la democracia en estos países. Rusia combatirá por la democracia - por su idea de democracia" (Krastev, 2005). Por tanto, mientras que en el interior del país se empezó a hablar de "democracia soberana” para reivindicar una soberanía que no está sujeta a interferencias externas y a la elaboración de un modelo político autónomo, incluso hacia el exterior se ha tratado de enviar un mensaje positivo diferente respecto al "imperialismo ideológico" que viene desde el Oeste (Kokoshin, 2006; Naumov y Slonov, 2007).

Para lograr este difícil objetivo, Moscú utiliza su principal recurso, la diáspora rusa, respecto a la cual en los últimos años el $\mathrm{Mi}$ nisterio de Asuntos Exteriores ha tomado diferentes iniciativas para fortalecer los vínculos entre la Federación y sus compatriotas que viven en el extranjero. En particular, en 2007 se ha creado la fundación Russkyj Mir (Mundo ruso), con la finalidad de difundir en el mundo la lengua y la cultura rusa, defender los derechos de las minorías de este país y apoyar la presencia de Rusia en los países vecinos (Kudors, 2010; Laruelle, 2015).

La fundación se ha encargado de coordinar la mayor parte de las organizaciones no gubernamentales que, después de las revoluciones de los colores, han adquirido una creciente importancia para influir sobre los procesos sociopolíticos en favor de Moscú.
De acuerdo con las principales teorías, que le reconocen al poder blando la posibilidad de fortalecer la autoridad de un país en un territorio a través su presencia cultural, la acción rusa se ha dirigido hacia esta dirección impulsando la difusión de centros culturales y el posible uso del ruso como "lengua franca" en las relaciones comerciales, culturales y científicas en el espacio eurasiático.

Sin embargo, no se puede ignorar la dificultad que ha encontrado Rusia en promover sus modelos culturales en territorios donde históricamente la presencia zarista antes y soviética después ha tomado la forma de un condicionamiento ideológico y de una dominación imperial. Por ejemplo, Moscú ha utilizado los intercambios culturales también en época soviética como parte de la ofensiva ideológica contra el expansionismo occidental. Uno de los casos más significativos ha sido la ex-Universidad Patricio Lumumba, ahora Universidad rusa de la Amistad de los pueblos, fundada en 1960 para ofrecer instrucción superior y formación profesional a jóvenes provenientes, en particular, de África, Asia y América Latina (Marchuk y Volociuk, 2010). El objetivo era, obviamente, formar una nueva generación de élites políticas amigas en áreas geográficas estratégicamente importantes en el periodo de la confrontación bipolar.

Por tanto, frente al recelo respecto a una actividad diplomática que recuerda estrategias de control pertenecientes al pasado, se destaca que los resultados obtenidos hasta el momento han sido bastante modestos. En general, el número de los rusos-hablantes que viven en el extranjero ha disminuido, y la presencia de las originarias escuelas rusas, situadas en los 
viejos países de la Unión Soviética, se ha reducido de manera significativa; asimismo, los programas de colaboración entre los miembros de la Comunidad de Estados Independientes (CEI) se ven afectados por la escasez de recursos que ofrecen becas menos atractivas respecto a las ofertas de Estados Unidos y la Unión Europea (Torkunov, 2013).

Por consiguiente, si por un lado Moscú sigue incrementando la difusión de su modelo educativo, por el otro trata de impulsar todas las actividades que permitan la exportación de productos de cultura de masas -como la música o la cinematografía- para conquistar a un más amplio público y reforzar una imagen positiva de Rusia.

Por ende, teniendo en cuenta el papel de los medios de comunicación en la formación de una favorable opinión pública internacional, en 2005 se creó el canal de noticias Russia Today (RT), que transmite en inglés, español y árabe en más de cien Estados; además, algunas de las más populares revistas, como Komsomolskaja Pravda y Argumenty i Fakty han empezado a publicar ediciones especiales en los países limítrofes (Ćwiek-Karpowicz, 2012, p. 7). Igualmente, diferentes canales de radio y de televisión han decidido transmitir en otros idiomas, y varios sitios rusos en Internet han adicionado al tradicional contenido en el idioma nacional, una sección en inglés para superar la barrera lingüística. El mismo Ministerio de Asuntos Exteriores ha transformado, desde 2011, su sitio para hacerlo más atractivo y, desde 2012, ha lanzado su canal en Youtube y abierto una cuenta en Twitter para acercarse de manera más directa a la gente (Shakirov, 2013).
Es indudable que la progresiva atención hacia estas herramientas ha sido determinada también por el contexto internacional. En particular, este ulterior empuje a la comunicación tecnológica está estrechamente relacionado con la guerra en Georgia de 2008, en el curso de la cual se evidenció la disparidad de los medios de comunicación de la propaganda occidental respecto a la rusa. De hecho, la intervención militar y el uso de la fuerza por parte de Moscú han representado un serio daño a la reputación rusa, en particular en el espacio pos-soviético, en la medida en que los países de la CEI no han reconocido la independencia de Abjasia y de Osetia del Sur temiendo que incidentes similares puedan afectar también su integridad territorial.

Frente a la perplejidad suscitada por una conducta que sigue utilizando un poder duro para afirmar su visión de las relaciones internacionales, la diplomacia rusa ha tratado, entonces, de recuperar su imagen y de empeñarse en fortalecer los vínculos con los viejos aliados.

Desde esta perspectiva, por un lado se han reconocido las potencialidades de Internet para una comunicación más inmediata y, por el otro, se ha actuado de manera más concreta potenciando las organizaciones dirigidas a reforzar las relaciones con la diáspora rusa presente en el exterior. Fue así como pocas semanas después del conflicto, en septiembre de 2008, se decidió fundar la Agencia Federal para la Comunidad de Estados Independientes, Compatriotas Residentes en el Extranjero y de Cooperación Humanitaria Internacional (Rossotrudničestvo), que tiene representaciones en ochenta países, a través de las cuales se fo- 
menta el conocimiento de una nueva Rusia, de su potencial económico, cultural y espiritual, y de los contenidos de su política interior y exterior. Además, en mayo de 2011 se creó el "Fondo de apoyo y protección de los derechos de los compatriotas rusos en el exterior", con el cual el Gobierno ruso se encarga de asistir a todos sus connacionales presentes en el exterior y de apoyarlos en caso de violaciones, para tutelar sus derechos e intereses legítimos (Ćwiek-Karpowicz, 2012, p. 8).

Es importante resaltar que las autoridades rusas han llegado a una definición bastante extensa de "compatriota" que incluye, no solo los ciudadanos de la Federación que viven afuera de los confines nacionales, sino también los habitantes de la vieja Unión Soviética y "todos los ciudadanos extranjeros que admiran la cultura y la lengua rusa" (Ćwiek-Karpowicz, 2012, p. 8). Interpretación, esta última, que parece bastante discutible en la medida en que termina por legitimar una política intrusiva hacia toda la región circundante, y representa un potencial riesgo para la soberanía y la integridad territorial de los países menores.

\section{¿SOFT POWER O SOFT PROPAGANDA?}

De tal manera, se considera que el principal problema conectado con un auténtico ejercicio del soft power es la tentación de convertirlo en una "soft propaganda". A pesar de haber reconocido la importancia de utilizar todos los recursos, sean materiales o inmateriales, para rehabilitar la reputación de Rusia y competir con los países occidentales en el mismo ámbito, el Gobierno ruso sigue confundiendo su capacidad de atracción con la instauración de políticas de influencia, en particular en los países de la región.

La verdadera debilidad del poder blando del Kremlin reside en la fragilidad de la misma idea rusa. De forma similar lo afirmaba el entonces director de Rossotrudničestvo, cuando decía que Rusia "no puede exportar ningún modelo alternativo a los ofrecidos por China, Occidente o por el mundo islámico, porque todavía no ha desarrollado ningún tipo de modelo" (Kosachev, 2012). Para presentarse al exterior como un modelo político y socioeconómico exitoso, Moscú tendría que solucionar antes que todo sus problemas internos. Un país caracterizado por una corrupción generalizada, un declive demográfico, crisis económicas, una democracia ambigua y la ausencia de una idea nacional unificadora, no es muy creíble como líder carismático.

De todas formas, no se puede subestimar la importancia de elementos como la cercanía lingüística, la cultura común, la historia compartida, los vínculos económicos y la dependencia de los recursos energéticos rusos que representan indudablemente un punto de partida para legitimar la existencia de una unión privilegiada entre todos los países de la región eurasiática. Por consiguiente, en tiempos más recientes, el Gobierno ruso ha privilegiado una política exterior más asertiva para presentarse como representante de una "internacional conservadora", de un polo geopolítico que reivindica la defensa de la soberanía nacional contra las presiones externas, rechaza la imposición de un modelo único global y promueve un sistema alternativo de las relaciones internacionales, no necesariamente dominado por Estados Unidos. 
En esta perspectiva, la recién nacida Unión Económica Eurasiática ${ }^{2}$ también representa la última etapa de los continuos esfuerzos para promover proyectos de integración en esta área geográfica y afirmarse como potencia líder en la región. Sin embargo, el mayor desafío de la política exterior rusa radica en la capacidad de modernizar el país y combinar sus intereses con los de los Estados socios para crear un entorno de cooperación favorable para todos. A fin de alcanzar estos objetivos, es necesario mejorar los instrumentos de un poder blando que permita limpiar su imagen internacional e incrementar la confianza en proyectos de integración que no se traduzcan en asimilación; convencer a las viejas repúblicas soviéticas de que Moscú no está buscando tanto ampliaciones territoriales, sino nuevas formas de colaboración que representen un beneficio real para todos los participantes.

Este proyecto puede convertirse en realidad solo abandonando las viejas lógicas de la contraposición del pasado siglo. Infortunadamente, los intentos rusos de desarrollar un poder blando y atractivo hacia el exterior se ven frustrados frente a crisis internacionales en las cuales Moscú regresa a las tradicionales manifestaciones de fuerza militar y chantaje económico.

Es natural, en este sentido, hacer referencia a la crisis ucraniana de 2014 que demostró, una vez más, cómo el uso tradicional de la fuerza refleja una política miope y anacróni$\mathrm{ca}$, que sirve a los intereses nacionales a corto plazo, pero destruye la posibilidad de llegar a resultados importantes. El principal obstáculo para cualquier proyecto de integración sigue siendo la asimetría de poder entre los diferentes Estados miembros y la desconfianza de las viejas repúblicas soviéticas respecto a vagas promesas de beneficios para todos y que, en el momento de la verdad, se revelan un instrumento más para regresar a la vieja política imperial rusa.

Indudablemente, Rusia podía contar con un verdadero soft power en Crimea, que tiene sus raíces en vínculos históricos y en la considerable presencia de una población de etnia rusa; sin embargo, frente a las dificultades con Kiev, Moscú no ha dudado en recurrir a la fuerza militar logrando reconquistar a Crimea, pero perdiendo mucho en términos de capacidad de atracción. De hecho, el mensaje enviado tanto a los países amigos como a los enemigos, es que Rusia exige respecto y, si necesario, está dispuesta a afirmar su autoridad de manera coercitiva (Tafuro, 2014).

Los límites de la visión rusa se destacan también en el mismo concepto de soft power, que difiere significativamente de la definición occidental. Es decir que, mientras en Estados Unidos o en Europa el poder blando se con-

\footnotetext{
2 Se hace referencia a la unión económica, nacida el primero de enero de 2015, que cuenta actualmente con la participación de Rusia, Kazajistán, Bielorrusia, Armenia y Kirguistán. La organización tiene el objetivo de garantizar la libre circulación de mercancías, servicios, capitales y mano de obra, y de coordinar políticas comunes en los sectores económicos estratégicos de energía, industria, agricultura y transporte (Scocozza, 2015).
} 
sidera como producto de una sociedad civil pluralista, de la cultura y, en general, de un estilo de vida, en Rusia sigue siendo el Kremlin el principal productor de soft power.

Si es verdad que también los gobiernos pueden promover la capacidad de atracción de sus países a través de diferentes medios, en el caso de Rusia se destaca una preminencia excesiva del centro, un soft power considerado como un instrumento de la política exterior del Estado y no como una consecuencia de la acción de la sociedad civil (Nikitina, 2014, p. 31).

En otras palabras, el verdadero problema de Rusia sigue siendo su credibilidad. Por tanto, es necesario definir claramente un sistema de valores sobre el cual se base la política del país, permitir un cierto nivel de previsión de sus acciones para que no dependan tanto del jefe de Estado en un preciso momento histórico, sino que sean el resultado de principios compartidos en una sociedad madura. La cuestión no se tiene que enfocar tanto en promover la imagen del país, la cultura o los valores de referencia - noticias que de todas formas circulan de manera suficiente- como en el nivel de confiabilidad de estas informaciones. Si provienen del Gobierno, pierden mucha credibilidad. "La mejor propaganda es la no propaganda" (Nye, 2013). La tarea de promoción del país, que en Occidente se encomienda en particular a las fundaciones, universidades y organizaciones de diferente tipo, en Rusia sigue siendo monopolio del Estado, lo que contribuye a la percepción de que esta sea una versión actualizada de la vieja propaganda soviética dirigida a la defensa de los intereses nacionales.

\section{CONCLUSIONES}

A la luz de estas consideraciones, el soft power ruso corresponde a una "peculiar combinación de una ideología de exclusividad nacional promovida por el Estado, manipulación de los símbolos culturales y nostalgia por los días felices de la Unión Soviética, una genuina atracción económica y política rusa de los países menos desarrollados de la Comunidad de los Estados Independientes y una amenaza externa publicitada por los medios de comunicación del Estado ruso" (Liu y Guan, 2014, p. 79).

Sin duda alguna, el actual contexto internacional no ha representado una oportunidad para solucionar las objetivas dificultades rusas. A pesar de los esfuerzos cumplidos también con ocasión de las Olimpiadas invernales de Sochi, en febrero de 2014, para difundir una imagen exitosa, el casi contemporáneo conflicto en Ucrania, el deterioro de las relaciones con Occidente, la caída del rublo han representado todos factores que han condicionado la reflexión del gobierno sobre las más oportunas estrategias diplomáticas.

En este contexto, la necesidad de presentarse ante la escena internacional a la conquista de nuevos socios ha tomado un enfoque competitivo, y no cooperativo, con los otros actores occidentales. Siendo realistas, es muy difícil hacer previsiones positivas con respecto a la eficacia del actual soft power ruso. De todas formas, las exitosas políticas de los países que han sabido utilizarlo representan un importante impulso para la Federación Rusa, que seguirá implementando las estrategias oportunas para ampliar su nivel de atracción. 
Además, la actual crisis internacional representa una oportunidad para replantear el enfoque adoptado hasta ahora coordinando nuevos mecanismos y ampliando, como es deseable, el número de los varios actores involucrados en el proceso. Solo de esta manera se podría imaginar a largo plazo un oportuno ejercicio de poder blando que convierta a Rusia en una potencia carismática, líder en Eurasia, y capaz de ofrecer su contribución a la nueva arquitectura del sistema internacional del siglo XXI.

\section{REFERENCIAS}

Ćwiek-Karpowicz, J. (2012). Limits to Russian Soft Power in the Post-Soviet Area. DGAP analyse, $8,1-11$.

Karabeshkin, L. y Sergunin, A. (2015). Understanding Russia's Soft Power Strategy.Politics, 3-4, 347-363.

Kokoshin, A. (2006). Russian Sovereignty and Sovereign Democracy. Russia in Global Affairs, 150-181.

Kosachev, K. (2012). The Specifics of Russian Soft Power. Russia in Global Affairs. Recuperado de http://eng.globalaffairs.ru/number/The-Specifics-of-Russian-Soft-Power-15683

Krastev, I. (2005). Russia’s Post-Orange Empire. Opendemocracy. Recuperado de http://www.opendemocracy.net/democracy-europe_constitution/ postorange_2947.jsp.

Kudors, A. (2010). "Russian World" - Russia’s Soft Power Approach to Compatriots Policy. Russian Analytical Digest, 81, 2-6.

Laruelle, M. (2015). The "Russian World". Russia's Soft Power and Geopolitical Imagination. Washington DC: Center on Global Interests.
Liu, Y. y Guan, H. (2014). The adjustment of soft power policy during the new-period Russian diplomatic transformation. Northeast Asia Forum, 24, 73-86.

Marchuk, N. y Volociuk, O. (2010). URAP y América Latina - 50 años de amistad. Moscú: Universidad de Rusia de la Amistad de los Pueblos.

Naumov, S. y Slonov, N. (2007). Suverennaja Democratija (democracia soberana). Svobodnaja Mysl, 3, 21-30.

Nikitina, Y. (2014). Rossijskij podxod k prodviženiju "mjagkoj sily" v sfere vnešnej politiki (Un acercamiento ruso al "poder blando" en el ámbito de la política exterior). Vestnik mGimo UNiversiteta, 2, 30-35.

Nye, J. (1990). Soft Power. Foreign Policy, 80, 153-171.

Nye, J. (2013). What Russia and China Don't Get About Soft Power. Foreign Policy. Recuperado de http:// foreignpolicy.com/2013/04/29/what-china-andrussia-dont-get-about-soft-power

Ó Beacháin, D. y Polese, A. (2012). The Colour Revolutions in the Former Soviet Republics. Successes and Failures. Abingdon y New York: Routledge.

Putin, V. (2012a). Rossija i menjajuščijcja mir (Rusia y el mundo que cambia). Moskovskie Novosti. Recuperado de http://www.mn.ru/politics/78738

Putin, V. (2012b). Soveščanie poslov i postojannych predstavitelej Rossii (Encuentro con los embajadores y representantes permanentes de Rusia). Recuperado de http://www.kremlin.ru/transcripts/15902.

Putin, V. (2013). Koncepcija vnešnej politiki Rossijskoj Federacii (Concepto de politica exterior de la Federación Rusa). Recuperado de http://www.mid. ru/brp_4.nsf/newsline/6D84DDEDEDBF7DA 644257B160051BF7F.

Scocozza, C. (2015). Modelos alternativos de integración económica: el caso de la Unión Económica Eurasiática. En La arquitectura del ordenamiento 
internacionaly su desarrollo en materia económica (pp. 463-489). Bogotá: Universidad Externado de Colombia.

Shakirov, O. (2013). Russian Soft Power Under Construction. Recuperado de http://www.e-ir.info/2013/02/14/russian-soft-power-under-construction.

Tafuro, E. (2014). Fatal attraction? Russia’s soft power in its neighborhood - Analyses. Eurasia Re- view. Recuperado de http://fride.org/download/29.05.2014_EurasiaReview_Us_ET.pdf Torkunov, A. (2013). Education as a Soft Power Instrument of Russia's Foreign Policy. Recuperado de http:// russiancouncil.ru/en/inner/?id_4=1495\#topcontent.

Tsygankov, A. P. (2006). If not by Tanks, then by Banks? The Role of Soft Power in Putin's Foreign Policy. Europe-Asia Studies, 7, 1079-1099. 